\title{
Spectral Doppler Waveform Analysis of Common Femoral Veins for the Detection of Right Ventricular Dysfunction in Acute Pulmonary Embolism
}

Bettina-Maria Taute ${ }^{{ }^{\star}}$, Hendrik Schmidt ${ }^{2}$, Andreas Gunter Bach ${ }^{3}$, Ronald Fischer ${ }^{1}$, Christin Le Hoa Tran ${ }^{1}$, Mroawan Amoury ${ }^{4}$ and Hannes Melnyk ${ }^{1}$

${ }^{1}$ University Hospital of the Martin-Luther-University Halle-Wittenberg, Department of Internal Medicine III/Angiology, Ernst-Grube-Str. 40, D-06120 Halle (Saale), Germany

${ }^{2}$ Hospital Magdeburg, Clinic of Cardiology, Birkenallee 34, D-39130 Magdeburg, Germany

${ }^{3}$ University Hospital of the Martin-Luther-University Halle-Wittenberg, Department of Radiology, Ernst-Grube-Str. 40, D-06120 Halle (Saale), Germany

${ }^{4}$ University Hospital of the Martin-Luther-University Halle-Wittenberg, Emergency Department, Ernst-Grube-Str. 40, D-06120 Halle (Saale), Germany

*Corresponding author: Bettina-Maria Taute, University Hospital of the Martin-Luther-University of Halle-Wittenberg, Department of Internal Medicine III / Angiology, Ernst-Grube-Str. 40, D-06120 Halle (Saale) Germany, Tel: +49 345557 3351; E-mail: bettina-maria.taute@uk-halle.de

Received November 12, 2014; Accepted December 29, 2014; Published December 30, 2014

Copyright: () 2015 Taute BM, et al. This is an open-access article distributed under the terms of the Creative Commons Attribution License, which permits unrestricted use, distribution, and reproduction in any medium, provided the original author and source are credited.

\begin{abstract}
Background

Changes of central and peripheral venous hemodynamics $(\mathrm{PVH})$ are registered in patients with acute pulmonary embolism (PE) with right ventricular dysfunction (RVD). It can be assumed the change of PVH is detectable through spectral Doppler waveform analysis of the common femoral veins (CFV) and regular respiratory modulated (RM) flow is replaced as a function of the severity of the RVD by cardiac modulation (CM) of the velocity-time spectra. The aim of this study is to examine, through detection of CM at the CFV, whether indirect diagnostics of the RVD is possible in patients with acute PE.
\end{abstract}

\section{Methods}

Quantification of RVD was performed through an echocardiographic right heart score (RHS) in patients with acute PE. The RHS integrated four parameters: the size of the right ventricle, the RV kinetics, the motion of the interventricular septum and the right atrial size. The sonographic quantification of RM and CM at the CFV was performed through measurement of the flow duration directed towards the heart in the velocity-time spectrum. The results of the RHS and the respective spectral Doppler waveform at the CFV were comparatively analysed.

\section{Results}

In 47 acute PE-patients CM was found in $53.2 \%$. A RHS of 1 was present in $17 \%$ and there was no CM. CM was found in $19 \%$ of patients with a RHS of 1.25 , and in $92 \%$ of patients with a RHS of 1.5 . All patients with a RHS of $\geq$ 1.75 had a CM. The sensitivity and specificity for the CM with a cut-off RHS of 1.5 amounted to 0.96 and 0.88 respectively.

\section{Conclusion}

The CM at the CFV is an indirect diagnostic criterion of RVD with acute PE. In the case of suspected PE, the absence of CM excludes a RVD. Disappearance of CM during the clinical course of PE indicates improvement in RV function.

Keywords: Pulmonary embolism; Right ventricular dysfunction; Echocardiographic right heart score; Peripheral venous hemodynamics; Ultrasound-spectral Doppler waveform; Common femoral vein

\section{Introduction}

After myocardial infarction and stroke, venous thromboembolism is the third most frequent cardiovascular cause of death. Guidelinecompliant diagnostic and therapeutic strategies can serve to improve the prognosis in cases of suspected or acute pulmonary embolism (PE). This includes the initial risk stratification, which differentiates into hemodynamically unstable and hemodynamically stable patients with PE. Hemodynamic instability is associated with severe right ventricular dysfunction (RVD), however hemodynamically stable patients can also present with different severity levels of RVD [1,2].

An acute pulmonary arterial thromboembolic obstruction with sudden increase of the pulmonal arterial pressure, dependent on severity level, leads to RVD with increase of the right ventricular wall stress, dilatation, acute tricuspid regurgitation, reduced right ventricular ejection and subsequently, possibility of reduced filling of the left ventricle. At significantly reduced ejection output of the left ventricle, hypotension and systemic and/or coronary hypoperfusion 
Citation: Taute BM, Schmidt H, Bach AG, Fischer R, Tran CLH, et al. (2015) Spectral Doppler Waveform Analysis of Common Femoral Veins for the Detection of Right Ventricular Dysfunction in Acute Pulmonary Embolism. J Cardiovasc Dis Diagn 3: 187. doi: 10.4172/2329-9517.1000187

Page 2 of 5

with myocardial ischemia will result, still aggravating the RVD [3,4]. Consequently, changes in the hemodynamics of central veins are found, which can persist up to the peripheral venous circulation. The effects of an acute pulmonary arterial obstruction of the heart and central veins can be detected by CT angiography and by echocardiogram [5-7]. As a measure of the quantification of the severity level of RVD with acute PE, an echocardiographic right heart score (RHS) can be used. This includes the size of the right ventricle, the right ventricular kinetics, the movement of the interventricular septum and the right atrial size [8].

To describe the peripheral venous hemodynamics, ultrasonography with spectral Doppler waveform analysis of the common femoral veins (CFV) is most suitable. Normally, the venous reflow can be mapped in the velocity-time spectrum at the CFV as spontaneous and respirophasic flow. This respiratory modulated (RM) flow is caused through the respiration-dependent intra-abdominal and intrathoracic pressure changes, as well as the breathing-dependent diaphragmatic movements. In the expiration phase, this results in directed flow towards the heart that decreases in the inspiration phase up to an inspiratory flow stop.

With acute RVD as a result of PE, there will be an increased filling of the entire venous reflux area. Intravascular pressure rises, prevents the physiological collapse of the inferior vena cava and lifts the respiratory dependence of the venous reflow. In the velocity-time spectrum of the CFV, the altered image of pulse-synchronous flow appears, which is known as cardiac modulation (CM) of the venous spectra. Here, it should be assumed that the change from respiratory to CM occurs as a function of the severity level of the RVD.

The aim of this study was to investigate whether, for patients with acute PE, the presence of CM in the spectral Doppler of the CFV correlates with the echocardiographically quantified severity level of acute RVD.

\section{Patients and Methods}

\section{Study design}

Patients with acute PE were enrolled in a prospective study. Evidence of PE was confirmed using chest computerised tomography angiography and radiographic images were analysed by experienced radiologists. After initiation of therapy, all patients were subjected as a matter of routine both to echocardiographic diagnosis with calculation of the RHS, performed by a cardiologist, and to duplex ultrasound for the diagnosis of lower-extremity deep vein thrombosis. Lowerextremity venous duplex ultrasound, performed by an angiologist, included a spectral Doppler waveform analysis of the CFV and made a distinction between a RM or a CM of the spectrum. The interval between the two examinations did not exceed six hours. In addition, diagnosticians were unaware of the results of the other study in each case. The exclusion criteria were hemodynamically unstability of patients, pelvic vein thrombosis or cava inferior vein thrombosis, and an interval of more than six hours between echocardiography and lower-extremity venous duplex ultrasound. All patients gave their informed consent.

\section{Echocardiographic RHS}

For the quantification of the hemodynamic severity level of acute $\mathrm{PE}$, an echocardiographic RHS was used that describes RVD in standardised and reproducible form [8]. Four echocardiographic parameters were integrated, where each parameter was evaluated in a point scale with the numbers 1 - normal, 2 - moderately disturbed or 3 - highly pathological. Through the calculation of the arithmetic mean from these four parameters, values result from one up to three points and steps of 0.25 points are possible (Table 1 ). With a RHS of 1 , there is no RVD, and RHS values $\geq 2.25$ indicate a clinically and prognostically relevant RVD [9].

\begin{tabular}{|c|c|c|c|}
\hline Score Parameter & Evaluation 1 & Evaluation 2 & Evaluation 3 \\
\hline $\begin{array}{l}\text { Right ventricular size } \\
\text { Visual assessment and } \\
\text { measurement }(\mathrm{mm}) \text { in the } \\
\text { apical four-chamber view } \\
\text { (end diastolic level with } \\
\text { the peaks of the open } \\
\text { tricuspid leaflet) }\end{array}$ & $\begin{array}{l}\mathrm{RV}<\mathrm{LV} \text { and } \\
\mathrm{RV}<30\end{array}$ & $\begin{array}{l}\text { Neither the } \\
\text { criteria for } \\
\text { evaluation } 1 \\
\text { still for } \\
\text { evaluation } 3 \\
\text { are fulfilled }\end{array}$ & $\begin{array}{l}R V>L V \text { and } \\
R V \geq 40\end{array}$ \\
\hline $\begin{array}{l}\text { Right ventricular kinetics } \\
\text { Visual assessment in the } \\
\text { apical four-chamber view }\end{array}$ & $\begin{array}{l}\text { In all segments } \\
\text { normokinetic }\end{array}$ & $\begin{array}{l}\text { Basal and } \\
\text { medial } \\
\text { hypokinetic }\end{array}$ & $\begin{array}{l}\text { Basal and } \\
\text { medial akinetic }\end{array}$ \\
\hline $\begin{array}{l}\text { Movement of the } \\
\text { interventricular septum } \\
\text { Visual assessment in the } \\
\text { parasternal section with } \\
\text { the involvement of } \\
\text { M-Mode }\end{array}$ & $\begin{array}{l}\text { In systole to } \\
\text { the LV }\end{array}$ & $\begin{array}{l}\text { In systole } \\
\text { partially to the } \\
\text { RV }\end{array}$ & $\begin{array}{l}\text { In systole } \\
\text { completely to } \\
\text { the RV }\end{array}$ \\
\hline $\begin{array}{l}\text { Right atrial size } \\
\text { Visual assessment in the } \\
\text { apical four-chamber view }\end{array}$ & $\mathrm{RA} \leq \mathrm{LA}$ & $\mathrm{RA}>\mathrm{LA}$ & $\begin{array}{l}\mathrm{RA}>\mathrm{LA} \\
\mathrm{RA} \text { a lot } \\
\text { greater than } \mathrm{LA}\end{array}$ \\
\hline
\end{tabular}

Table 1: Definition and evaluation of the echocardiographic right heart score [8]

\section{Spectral Doppler waveform analysis of CFV}

Respiratory and cardiac modulation of the velocity-time spectrum at the CFV can be rapidly differentiated from each other qualitatively. Nevertheless, quantitative differentiation was aimed for in a preliminary study. This was achieved through measurement of the flow duration directed towards the heart in the velocity-time spectrum of the CFV in 36 subjects without cardiac or pulmonary pre-existing conditions and in 37 patients with right heart failure of different genesis (e.g., congestive heart failure, $\mathrm{PE}$, chronic pulmonary arterial hypertension). Patients with right heart failure showed $\mathrm{CM}$ and subjects without cardiac or pulmonary diseases a RM in the velocitytime spectrum of the CFV. A mean period length was found for RM of $3.08 \pm 0.58 \mathrm{sec}$ ( $\operatorname{span} 2.08-4.61 \mathrm{sec}$ ). For CM, a mean period length could be calculated of $0.87 \pm 0.18$ sec. (span $0.53-1.29 \mathrm{sec}$ ). The difference was found to be significant $(\mathrm{p}<0.0001)$. From this, the definition followed that $\mathrm{CM}$ is present when the period length is less than two seconds and accordingly a RM of the Doppler spectrum is characterised by a period length of at least two seconds.

For the current study, a representation of the right and left CFV was standardised in longitudinal section, with a derivative of the velocitytime spectrum immediately distal to the junction of the great saphenous vein (Sonoline Antares Siemens Healthcare, Erlangen). According to qualitative criteria and the named quantitative criteria, a division was made in patients with respiratory or cardiac spectral Doppler waveforms. Figure 1 indicates the RM and Figure 2 the CM with the measurement of the period length in the velocity-time spectrum of the CFV. 
Citation: Taute BM, Schmidt H, Bach AG, Fischer R, Tran CLH, et al. (2015) Spectral Doppler Waveform Analysis of Common Femoral Veins for the Detection of Right Ventricular Dysfunction in Acute Pulmonary Embolism. J Cardiovasc Dis Diagn 3: 187. doi: $10.4172 / 2329-9517.1000187$

Page 3 of 5

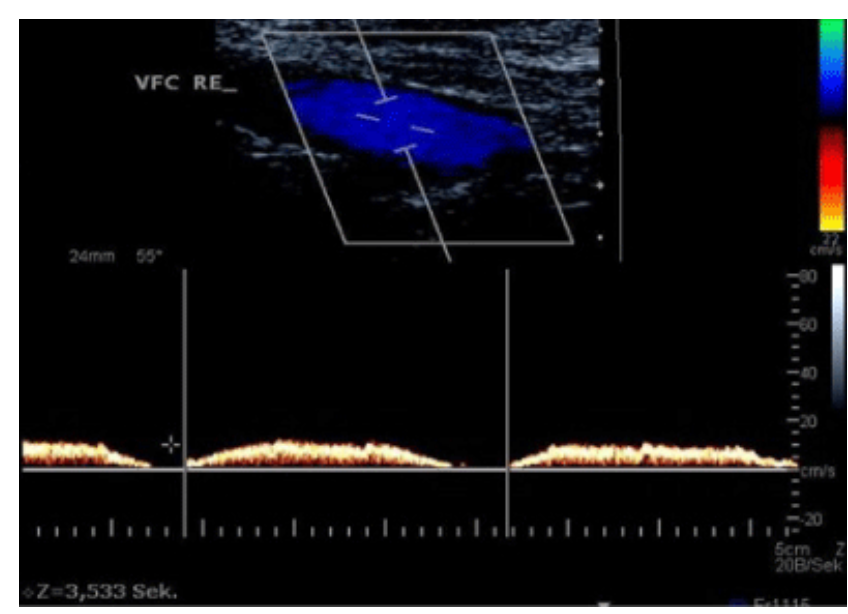

Figure 1: Colour-flow Doppler and pulsed Doppler spectral waveform at the right common femoral vein with respiratory modulation in a healthy subject. Note the flow directed towards the heart in the expiratory phase (positive deflection) and of the flowstop discernible in the inspiratory phase. The vertical lines delimit a respiratory-mediated flow period of 3.5 seconds duration.

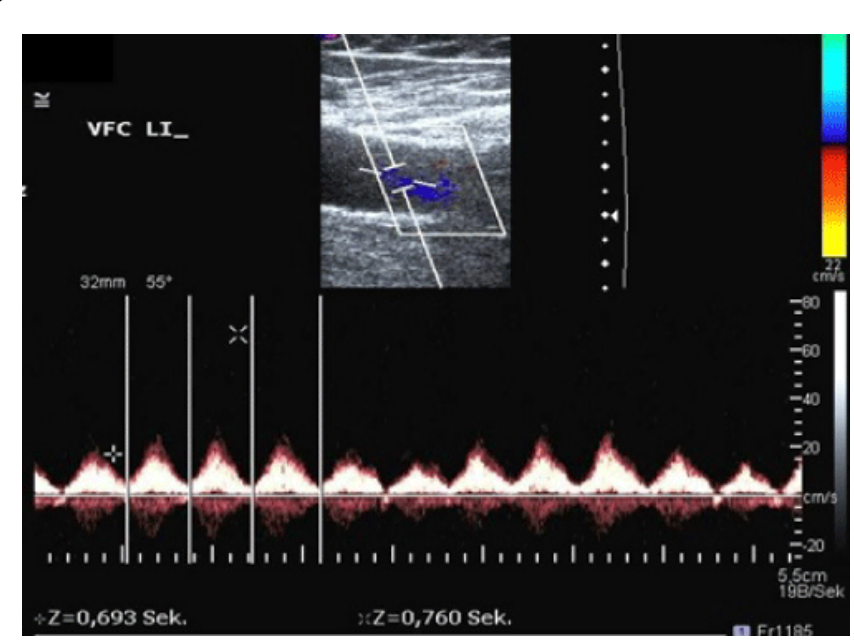

Figure 2: Colour-flow Doppler and pulsed Doppler spectral waveform at the left common femoral vein with cardiac modulation in a patient with acute pulmonary embolism. The respiratory modulation is completely reversed. The vertical lines delimit a flow period directed towards the heart. The period length amounts to approximately 0.7 seconds.

\section{Follow-up studies in patients with acute PE}

Some PE patients who had been characterised initially with spectral Doppler waveform analysis and echocardiographically by means of RHS received an echocardiographic and colour duplex sonographic follow-up, again time synchronised. The results of the RHS and the corresponding spectral Doppler waveform at the CFV were evaluated to detect the regression of RVD under PE therapy.

\section{Statistical analysis}

The results of the calculation of the RHS and of the spectral Doppler waveform analysis of the CFV were correlated with each other, a receiver operating characteristics (ROC) analysis was carried out to test the effectiveness of CM to predict RVD and sensitivity and specificity were calculated. For the statistical analysis, SPSS Version 22.0 was used.

\section{Results}

Correlation of echocardiographic RHS and spectral Doppler waveform of the $\mathrm{CFV}$ in acute $\mathrm{PE}$

In 47 patients with acute PE (55.3\% women, $44.7 \%$ men; mean age 59.81 years), the RHS and spectral Doppler waveform of the CFV were analysed with regard to the presence of RM or CM. The results are listed in Table 2. A RHS of 1 was present in $17 \%(n=8)$ of patients. None of these patients had CM in the velocity-time spectrum of the CFV. CM was found in $19 \%$ of patients with a RHS of $1.25(34 \%$, $\mathrm{n}=16)$, and $92 \%$ of patients had CM with a RHS of $1.5(28 \%, \mathrm{n}=13)$. All patients with a RHS of $1.75-2.5(21 \%, n=10)$ showed CM.

\begin{tabular}{|l|l|l|l|}
\hline \multirow{2}{*}{ Patients } & \multirow{2}{*}{ Right heart score } & $\begin{array}{l}\text { Respiratory } \\
\text { modulation }\end{array}$ & $\begin{array}{l}\text { Cardiac } \\
\text { modulation }\end{array}$ \\
\cline { 3 - 4 } & & $\mathbf{n}(\%)$ & $\mathbf{n}(\%)$ \\
\hline 8 & 1 & $8(100)$ & 0 \\
\hline 16 & 1.25 & $13(81.25)$ & $3(18.75)$ \\
\hline 13 & 1.5 & $1(7.69)$ & $12(92.31)$ \\
\hline 1 & 1.75 & 0 & $1(100)$ \\
\hline 3 & 2 & 0 & $3(100)$ \\
\hline 5 & 2.25 & 0 & $5(100)$ \\
\hline 1 & 2.5 & 0 & $1(100)$ \\
\hline
\end{tabular}

Table 2: Echocardiographic right heart score and corresponding spectral Doppler waveform at the common femoral veins in 47 patients with acute pulmonary embolism

ROC analysis was used to evaluate RHS levels with regards to the presence or absence of CM. The area under the curve (AUC) was calculated to be 0.94 with $\mathrm{p}<0.0001$, a standard error of 0.031 and an asymptotic confidence interval (95\%) ranging from 0.88 to 1 . The sensitivity and specificity for the CM with a RHS cut-off of 1.5 amounted to 0.96 and 0.88 respectively. Figure 3 shows the ROC curve.

\section{Follow-up studies in patients with acute PE}

For six patients with acute PE, both initially and in the follow-up, the RHS and the waveform were recorded in the velocity-time spectrum of the CFV (Table 3). 
Citation: Taute BM, Schmidt H, Bach AG, Fischer R, Tran CLH, et al. (2015) Spectral Doppler Waveform Analysis of Common Femoral Veins for the Detection of Right Ventricular Dysfunction in Acute Pulmonary Embolism. J Cardiovasc Dis Diagn 3: 187. doi: $10.4172 / 2329-9517.1000187$

Page 4 of 5

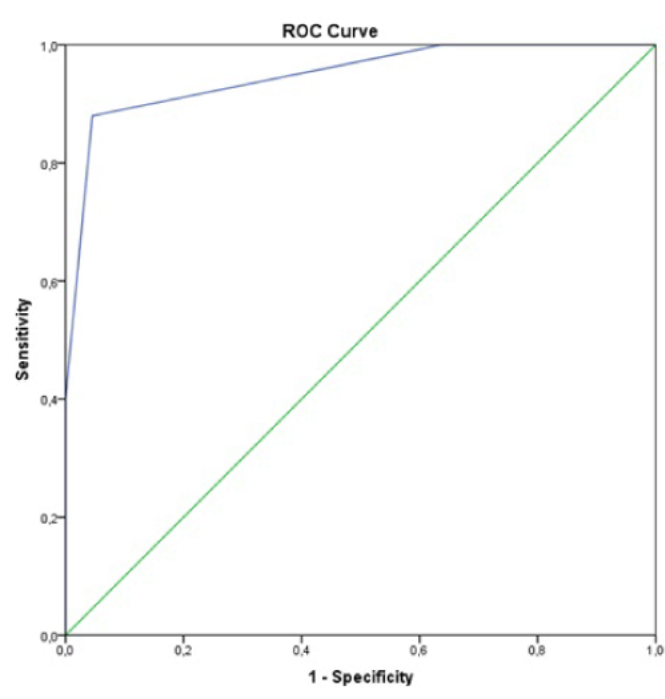

Figure 3: The receiver operating characteristics (ROC) curve for right heart score levels in patients with acute pulmonary embolism with cardiac modulation (present vs. absent) as the variable and assumption of nonparametric data distribution. Blue line - cardiac modulation, green line - reference line

\begin{tabular}{|c|c|c|c|c|}
\hline Patient & Age / sex & Day & RHS & CM /RM \\
\hline \multirow{2}{*}{1} & \multirow{2}{*}{$67 / f$} & 1 & 1.75 & $\mathrm{CM}$ \\
\hline & & 14 & 1 & $\mathrm{RM}$ \\
\hline \multirow{2}{*}{2} & \multirow{2}{*}{$69 /$ ภ } & 1 & 2 & $\mathrm{CM}$ \\
\hline & & 6 & 1 & $\mathrm{RM}$ \\
\hline \multirow{2}{*}{3} & \multirow{2}{*}{$81 / q$} & 1 & 2.5 & $\mathrm{CM}$ \\
\hline & & 6 & 1.5 & $\mathrm{CM}$ \\
\hline \multirow{2}{*}{4} & \multirow{2}{*}{$72 / q$} & 1 & 2.25 & $\mathrm{CM}$ \\
\hline & & 5 & 1.25 & RM \\
\hline \multirow{3}{*}{5} & \multirow{3}{*}{$65 / 9$} & 1 & 1.75 & $\mathrm{CM}$ \\
\hline & & 3 & 1.5 & $\mathrm{CM}$ \\
\hline & & 16 & 1 & RM \\
\hline \multirow{2}{*}{6} & \multirow{2}{*}{$59 /$ ภ } & 1 & 1.5 & $\mathrm{CM}$ \\
\hline & & 7 & 1 & $\mathrm{RM}$ \\
\hline
\end{tabular}

Table 3: Echocardiographic right heart score and corresponding spectral Doppler waveform at the common femoral veins in the clinical course of six patients with acute pulmonary embolism

\section{Discussion}

As an essential result of this study, it could be shown that RVD with acute PE influenced the peripheral venous hemodynamics. The quantitative analysis of the RVD by means of echocardiographic RHS proved that in the absence of RVD, corresponding to RHS $=1$, no change of RM of the velocity-time spectrum of the CFV occurred. Already, a low RVD (RHS $=1.25$ ) led, in a few cases, to the change of the spectral waveform of the CFV, with change from RM into CM. All patients with a RHS $\geq 1.75$ exclusively presented with $\mathrm{CM}$. The sensitivity and specificity for the presence of $\mathrm{CM}$, calculated for the cut-off of the RHS of 1.5 , was 0.96 and 0.88 respectively.

It has to be derived that, in normotensive patients with acute PE, a regular RM of the velocity-time spectrum at the CFV excludes RVD and hence identifies PE patients with a low risk of short-term mortality. For PE patients who can be assigned to this low-risk group, there is the option of an early discharge from hospital [10]. The assessment of the CFV is done in to evaluate the iliofemoral veins as a source of thromboembolism, additional evaluation for spectral flow takes only a minute more.

If $\mathrm{PE}$ is suspected in a hemodynamically stable patient in the emergency department, the reported technique can be used to rapidly exclude CM. If CM is detected, the patient can be triaged promptly to a definitive diagnosis by means of contrast-enhanced chest computerised tomography angiography.

Another advantage of the method is in the follow-up monitoring of patients with PE. The regression of the RVD becomes easily trackable with the presented method and costlier techniques for the same purpose can be avoided. Moreover, at the end of the regular treatment duration of 3-6 months after venous thromboembolism, which includes the documentation of still present venous pathologies of the leg veins, an additional assessment of the spectral Doppler waveform of the CFV can provide information as to whether pre-existing RVD has completely regressed. If CM is detected, there is a possibility that a chronic pulmonal arterial hypertension has developed and should be considered in the differential diagnosis. If this is the case, further diagnostics are required.

The Doppler spectrum waveform analysis of the CFV can be considered as a rapid and safe procedure, which is able to exclude hemodynamically relevant RVD according to indirect criteria, provided there is no venous obstruction proximal to the level of interrogation. The method presented in this study can possibly attain significance in the emergency department in order to accelerate the PE diagnostic process in the differential diagnosis of dyspnoea and thorax pain. In this regard, further scientific evaluation is required.

\section{Limitations}

Sonographic analysis of the peripheral hemodynamics does not replace any other method in the acute diagnosis of PE. Restrictions of the methodology arise concerning patients with pre-existing cardiac or pulmonary disease. $\mathrm{CM}$ at the $\mathrm{CFV}$ is also described as associated with the following pathological conditions: congestive heart failure, right heart failure, elevated right atrial pressure and tricuspid regurgitation, COPD or chronic pulmonary arterial hypertension [11-16].

The method presented here is not applicable in cases of monophasic flow patterns or absent venous flow in the velocity-time spectrum of $\mathrm{CFV}$, and venous obstruction proximal to the CFV must be expected. 
Citation: Taute BM, Schmidt H, Bach AG, Fischer R, Tran CLH, et al. (2015) Spectral Doppler Waveform Analysis of Common Femoral Veins for the Detection of Right Ventricular Dysfunction in Acute Pulmonary Embolism. J Cardiovasc Dis Diagn 3: 187. doi: 10.4172/2329-9517.1000187

Page 5 of 5

\section{Conclusion}

RM and CM can be safely differentiated from each other in the velocity-time spectrum of the CFV. The severity level of RVD with acute PE, quantified with an echocardiographic RHS, correlates significantly with changes in the peripheral venous hemodynamics. The CM at the CFV is an indirect diagnostic criterion of RVD with acute PE. In the absence of CM in the Doppler spectrum of CFV, RVD can be excluded. Disappearance of CM during the clinical course of PE indicates improvement in right ventricular function. The reported method represents an important extension to the indirect assessment of right ventricular function, but it does not replace any other method in the acute diagnosis of PE.

\section{References}

1. Konstantinides S, Goldhaber SZ (2012) Pulmonary embolism: risk assessment and management. Eur Heart J 33: 3014-3022.

2. Cho JH, Kutti Sridharan G, Kim SH, Kaw R, Abburi T, et al. (2014) Right ventricular dysfunction as an echocardiographic prognostic factor in hemodynamically stable patients with acute pulmonary embolism: a meta-analysis. BMC Cardiovasc Disord 14: 64

3. Lualdi JC, Goldhaber SZ (1995) Right ventricular dysfunction after acute pulmonary embolism: pathophysiologic factors, detection, and therapeutic implications. Am Heart J 130: 1276-1282.

4. Kreit JW (2004) The impact of right ventricular dysfunction on the prognosis and therapy of normotensive patients with pulmonary embolism. Chest 125: 1539-1545.

5. Kang DK, Thilo C, Schoepf UJ, Barraza JM Jr, Nance JW Jr, et al. (2011) CT signs of right ventricular dysfunction: prognostic role in acute pulmonary embolism. JACC Cardiovasc Imaging 4: 841-849.
6. Aghajanzadeh D, Gassanov N, Schmidt M, Semmo N, Er F (2010) Imaging techniques for the diagnosis of pulmonary embolism. Reports in Medical Imaging 3: 129-139.

7. Goldhaber SZ (2002) Echocardiography in the management of pulmonary embolism. Ann Intern Med 136: 691-700.

8. Wacker P, Wacker R (2005) [Pulmonary embolism--current therapy]. Dtsch Med Wochenschr 130: 1697-1700.

9. Wacker P, Wacker R (2005) [Thrombolytic therapy in acute pulmonary embolism]. Herz 30: 261-268.

10. Chalikias G, Konstantinides S (2014) Acute phase treatment of pulmonary embolism. Curr Vasc Pharmacol 12: 393-400.

11. Selis JE, Kadakia S (2009) Venous Doppler sonography of the extremities: a window to pathology of the thorax, abdomen, and pelvis. Am J Roentgenol 193: 1446-1451.

12. Kakish ME, Abu-Yousef MM, Brown PB, Warnock NG, Barloon TJ, et al. (1996) Pulsatile lower limb venous Doppler flow: prevalence and value in cardiac disease diagnosis. J Ultrasound Med 15: 747-753.

13. Abu-Yousef MM, Kakish ME, Mufid M (1996) Pulsatile venous Doppler flow in lower limbs: highly indicative of elevated right atrium pressure. AJR Am J Roentgenol 167: 977-980.

14. Krahenbuhl B, Restellini A, Frangos A (1984) Peripheral venous pulsatility detected by Doppler method for diagnosis of right heart failure. Cardiology 71: 173-176.

15. McClure MJ, Kelly BE, Campbell NS, Blair PH (2000) Duplex Doppler ultrasonography of lower limb veins: detection of cardiac abnormalities. Clin Radiol 55: 533-536.

16. Abbas M, Yahya MM, Hamilton M, Mwipatayi BP, Sieunarine K (2005) Sonographic evaluation of chronic venous insufficiency in right heart failure. J Diagn Med Sonogr 21: 238-242. 